\title{
Immature Platelet Fraction (IPF) Levels in Acute Coronary Syndrome (ACS) Patients
}

\author{
Enna Berkah Sari ${ }^{1}$, Nizam Zikri Akbar², Herman Hariman ${ }^{1}$ \\ ${ }^{1}$ Department of Clinical Pathology, Faculty of Medicine, Universitas Sumatera Utara (USU) / Haji Adam Malik \\ Hospital Medan \\ ${ }^{2}$ Department Cardiovascular and Vascular Disease, Faculty of Medicine, Universitas Sumatera Utara (USU) / \\ Haji Adam Malik Hospital Medan \\ Corresponding Author: Enna Berkah Sari
}

\begin{abstract}
Background: Acute Coronary Syndrome (ACS) is a major cardiovascular problem because it causes high hospital admissions and mortality rates. Acute Coronary Syndrome is divided into 3 (three), namely: unstable angina pectoris (UAP), myocardial infarction without ST segment elevation (NSTEMI), and myocardial infarction with ST segment elevation (STEMI). In addition to changes in biomarkers of heart injury, the platelet index (IPF = immature platelet fraction) will also change the level difference between STEMI with NSTEMI/UAP. Objective: To determine the differences in IPF levels of ACS patients with STEMI and NSTEMI/UAP
\end{abstract}

Method: Observational analytic with cross sectional approach. The subjects of this study were 80 patients who came to the emergency installation of integrated heart center Emergency Room Haji Adam Malik Hospital Medan from May 2019 to September 2019 and was diagnosed with ACS (STEMI or NSTEMI/ UAP). The sample in the study was the patient's venous blood and put it in an EDTA tube, then immediately checked the IPF value/level using the automatic hematology analyzer. Patients with heart failure or patients with thrombocytopenia were not included in this study.

Results: In this study, the demographic characteristics of the ACS patients based on gender were male $77.5 \%$ STEMI and $87.5 \%$ NSTEMI/UAP while women $22.5 \%$ STEMI and $12.5 \%$ NSTEMI/UAP. The results of the STEMI patient's IPF levels Compared with
NSTEMI/UAP, the median is $6.2(3.5-16.8)$ VS $2.9(0.7-12)$ with a p-value of 0.0001 .

Conclusion: The characteristics of ACS patients based on the results of sex were that there were more men with NSTEMI/UAP than those with STEMI. There was a significant difference in the IPF levels of STEMI with NSTEMI/UAP.

Keywords: Immature Platelet Fraction (IPF), Acute Coronary Syndrome (ACS), ST-Segment Elevation Myocardial Infarction (STEMI), Non ST-Segment Elevation Myocardial Infarction (NSTEMI), Unstable Angina Pectoris (UAP).

\section{BACKGROUND}

Acute coronary syndrome (ACS) is a group of symptoms caused by an acute disruption of coronary artery blood flow, generally caused by narrowing of the coronary arteries due to atherosclerotic plaques which then experience a tear and this triggers the occurrence of blood clots (Ibanez B, 2017), and is a a major cardiovascular problem because it causes high hospital admissions and mortality rates (PERKI, 2018).

Acute coronary syndrome is a process that begins with atherosclerosis in the form of waxy plaques or crusts that cause total or sub-total narrowing and blockage of the coronary arteries, so that blood flow is not sufficient to drain the heart muscle and then symptoms of ischemia and damage to the heart muscle (necrosis / infarction). Plaque caused by narrowing of the coronary arteries can be mild to severe, 
and the criteria for treatment are not only determined by the current narrowing, but the location and type of narrowing (Rubini Gimenez M, 2014).

Platelets play a role in the pathogenesis of cardiovascular disease including atherosclerotic complications such as unstable angina, STEMI, and heart failure. Platelet hyperactivity and local platelet activation have been shown to play a role in acute coronary events. Platelet size is known to reflect platelet activity which is indirectly measured by parameters. Larger platelets have denser granules, are metabolically and enzymatically more active than normal platelets. The platelet index is related to the functional status of the platelets and is a risk marker that appears for atherothrombosis and it is logical if there is an increase in IPF (Obeidi SR, 2013).

Immature platelets are young platelets that have just been released from the bone marrow, and some data show that this subpopulation of platelets is different from other platelets. In particular, they are large and reactive, and previous research has indicated changes related to $\mathrm{COX}$ metabolism. It has been reported that immature newly formed platelets express more COX-2 resulting in increased thromboxane production and an increased risk of thrombus and the development of atherosclerotic lesions seems to be in line with increased activation of thrombopoiesis in which the cytoplasm maturation of megakaryocytes is faster than nucleus maturation, which comes from sized platelets. larger, produce more thromboxane A2 and show greater reactivity in the platelet aggregation curve (Costa SC, 2015).

Acute coronary syndromes are divided into: myocardial infarction with ST segment elevation $($ STEMI $=$ ST segment elevation myocardial infarction), myocardial infarction with non elevation with non ST segment elevation $($ NSTEMI $=$ Non $\mathrm{ST}$ segment elevation myocardial infarction) and unstable angina pectoris (UAP) (PERKI, 2018).
The risk factors for ACS are divided into two: reversible (hypertension, cholesterol, smoking, obesity, diabetes mellitus, life style) and irreversible (age, gender and disease history) (Pasupathy S,2015).

Management carried out on ACS patients is by looking at the results of clinical ECG examinations and recording ECG assessments and examination of cardiac enzyme results (PERKI 2018). And the therapies given include anti-ischemic, anticoagulant, antiplatelet, thrombolytic / fibrinolytic, and other supporting drugs such as ACE inhibitors to prevent remodeling and statin drugs for plaque stabilization (Rubini Gimenez M, 2014).

According to a study by Grove et al, IPF is increased in patients with ACS, especially in patients with STEMI. The mean geometrical [95\% confidence interval] IPF was 2.51 [2.04-3.10] in healthy subjects, 2.87 [2.45-3.36] in CAD patients, $2.93[2.72-3.15]$ in the non-STEMI / unstable angina group and 3.71 [3.45-3.99] in the patients with STEMI (ANOVA: $p$ $<0.0001)$. This difference remained significant after adjusting for baseline characteristics $(\mathrm{p}=0.0003)$. In active smokers, IPF was $18 \%$ higher than in nonsmokers ( $p=0.007)$, and IPF was $16 \%$ higher in diabetics compared to nondiabetics ( $p=0.060$ ) (Grove EL, Hvas AM, Kristensen SD, 2009). This finding is also consistent with the findings of Lakkis et al, who reported higher IPF in STEMI patients than in NSTEMI and UAP patients (Khalifa KA et al, 2017).

\section{METHOD}

Analytic observational using cross sectional approach. The sample in this study were all patients who came to emergency installation of an integrated heart center Hajj Adam Malik Hospital Medan and diagnosed with ACS which had been classified into STEMI with NSTEMI/UAP in May 2019 to September 2019. Then the patient was taken of venous blood and put into an EDTA tube and immediately checked the value / level 
of his IPF by using a hematology automatic analyzer (Sysmex XN-1000). Patients with heart failure or patients with thrombocytopenia were not included in this study.

\section{RESULT}

\section{Demographic Characteristics of Research Subjects}

A study that aims to determine the comparison of levels of Immature Platelet Fraction in the blood of patients with acute coronary syndrome STEMI type and type NSTEMI/UAP. The characteristics of the subjects in this study can be seen in table 1 below:

Table1. Demographic Characteristics of Research Subjects

\begin{tabular}{|c|c|c|c|c|c|c|}
\hline & & \multicolumn{2}{|c|}{ STEMI } & \multicolumn{2}{|c|}{ NSTEMI/UAP } & \multirow[t]{2}{*}{ p-value } \\
\hline & & $\mathbf{N}$ & $\%$ & $\mathbf{N}$ & $\%$ & \\
\hline \multirow[t]{2}{*}{ Jenis Kelamin } & Pria & 31 & 77.5 & 35 & 87.5 & \multirow[t]{2}{*}{0.37} \\
\hline & Wanita & 9 & 22.5 & 5 & 12.5 & \\
\hline \multirow[t]{5}{*}{ Usia } & $<40$ tahun & 4 & 10.0 & 6 & 15 & \multirow[t]{5}{*}{0.31} \\
\hline & $40-49$ tahun & 13 & 32.5 & 8 & 20 & \\
\hline & $50-59$ tahun & 10 & 25.0 & 14 & 35 & \\
\hline & $60-69$ tahun & 7 & 17.5 & 10 & 25 & \\
\hline & $\geq 70$ tahun & 6 & 15.0 & 2 & 5 & \\
\hline \multicolumn{2}{|l|}{ Jumlah } & 40 & 100.0 & 40 & 100 & \\
\hline
\end{tabular}

Of all study subjects, the majority of the sexes were male consisting of $77.5 \%$ of STEMI patients and $87.5 \%$ of NSTEMI/ UAP patients, while female sex was only $22.5 \%$ of STEMI patients and $12.5 \%$ of NSTEMI/UAP patients.

As for age, most of the patients were in the age range of $40-49$ years $(32.5 \%)$ in STEMI patients, and 50-59 years (35\%) in NSTEMI/UAP patients. In this study, $10 \%$ of patients under 40 years of age were found in the STEMI group and $15 \%$ in the NSTEMI/UAP group. The results of statistical analysis show that there is no relationship between sex and age with differences in the incidence of STEMI or NSTEMI/UAP.

\section{Differences in IPF Levels for STEMI and NSTEMI/UAP Patients}

The main objective of this study was to determine the difference in IPF levels between patients with acute coronary syndrome who experience STEMI and NSTEMI/UAP, as shown in Table 2:

Table 2. Comparison of IPF levels Sufferers from STEMI and NSTEMI/UAP

\begin{tabular}{|l|l|l|l|}
\hline & STEMI & NSTEMI/UAP & \multirow{2}{*}{ p-value } \\
\cline { 1 - 3 } & median(min - max) & median(min - max) & \\
\hline IPF (\%) & $6.25(3.5-16.8)$ & $2.95(0.7-12,0)$ & 0.0001 \\
\hline
\end{tabular}

*)The comparison test is using the Mann Whitney test because the data are not normally distributed
Based on table 2, it can be seen that the IPF level of patients with acute coronary syndrome who experience STEMI is much higher than those with acute coronary syndromes experienced NSTEMI/UAP, which is obtained median (min-max) 6.25 (3.5-16.8)\% vs $2.95(0.7-12.0) \%$ with a pvalue of 0.0001 .

\section{DISCUSSION}

\section{Demographic characteristics of research subjects}

A study has been conducted comparing the levels of Immature Platelet Fraction (IPF) in patients with acute coronary syndrome. This study involved 80 subjects, consisting of 40 people with STEMI (ST-elevation myocardial infarction) and 40 people with NSTEMI/ UAP (Non ST-elevation myocardial infarction / unstable angina pectoris).

Based on demographic characteristics, it was found that the largest gender of sufferers of acute coronary syndrome was male, both in STEMI patients $(77.5 \%)$ and NSTEMI/UAP patients $(87.5 \%)$. This finding is not much different from the results obtained by Paramita et al. in Makassar in 2019 which found that sufferers of acute coronary syndrome were 
dominated by male sex, reaching $73.1 \%$. This is consistent with the theory that men are a separate risk factor for acute coronary syndrome. Conversely, women of active reproductive age are more protected from the incidence of acute coronary syndrome because of the protective effect of the hormone estrogen. After women enter menopause, these protective factors will disappear, and the magnitude of the risk of this ACS incidence will be the same between men and women (Paramita et al., 2019).

Based on age characteristics, it was found that patients with acute coronary syndrome were predominantly aged 40-49 years in the STEMI group (32.5\%) and 5059 years old in the NSTEMI/UAP group (35\%). The study conducted by Paramita et al. also found a relatively similar thing where $82.1 \%$ of the incidence of acute coronary syndrome was found in the young adult age group (aged 40-60 years). Age over 40 years is a separate risk factor for acute coronary syndrome which is associated with degeneration, endothelial injury, and fat accumulation in coronary arteries and the formation of atherosclerotic plaques (Paramita et al., 2019).

However, it needs to be of particular concern, that in this study the incidence of acute coronary syndrome was found in subjects aged under 40 years, namely $10 \%$ in the STEMI group, and as many as $15 \%$ in the NSTEMI and UAP group. The existence of these findings confirms that in recent years, there has been a shift in the age of patients with acute coronary syndrome, where over time the incidence of ACS at under 40 years of age is common. An important implication of these findings is that greater efforts are needed to control the risk factors associated with acute coronary syndrome, particularly lifestyle-related modifiable risk factors.

The results of statistical test analysis using the Chi Square test, found that there was no significant relationship between sex and age with the type of acute coronary syndrome attack experienced, whether
STEMI or NSTEMI/UAP ( $\mathrm{p}>0.05$ ). This means that the variables age and sex are not confounding variables (confounding factors) in this study.

\section{Discussion of immature platelet fraction (IPF) Levels in Acute Coronary Syndrome (ACS)}

Acute coronary syndrome is an emergency with clinical manifestations of pain in the chest or a group of symptoms resulting from acute myocardial ischemia ranging from UAP to acute myocardial infarction (AMI). Myocardial infarction consists of STEMI and NSTEMI. The presence of ST segment elevation on the EKG reflects total occlusion of the coronary arteries. Meanwhile, in the case of NSTEMI and UAP, arterial occlusion occurs in part of the lumen of the blood vessels. Both of them have similar clinical and pathophysiological symptoms but differ in severity (Paramita et al., 2019).

Research has shown that measuring platelet activity can identify patients at risk for cardiovascular disease. However, the measurement of platelet activity to predict ACS has not been routinely used for diagnosis and decision making and is only used for research proposals.

Ex vivo measurements of platelet activity, which use only a few agonists to activate platelet aggregation, often do not reflect true in vivo activity. In addition, anticoagulants in blood collection tubes are known to affect the normal function of platelets by chelating calcium in plasma. Until now, the usefulness of platelet activity in predicting ACS is still in doubt because there is not enough data to support the benefits of examining platelet activity. In addition, the best cut-off value for identifying patients at risk of cardiovascular disease has not been determined (Huang HL et al., 2019).

Platelets have a central role in the pathogenesis of ACS. Acute coronary syndrome results from platelet plaques in the coronary arteries. Mean Platelet Volume (MPV) is a measure of the average platelet 
count in the blood and reflects platelet activation. Meanwhile, Reticulated platelets or IPF are young platelets that are released into the circulation and have potential as markers of platelet activation and turnover. The benefits of these two parameters have been examined in the case of SKA (Grove EL et al, 2019).

A study conducted by Erik et al in Denmark showed a significant increase in IPF in ACS patients. The study conducted by Marie et al. in the Netherlands demonstrated that IPF was a more sensitive and specific diagnostic marker earlier than MPV for evaluating platelet turnover in ACS patients and showed a positive correlation between MPV and IPF. The study conducted by Jose et al. in the United States shows that an increase in IPF within 24 hours indicates a poor prognosis, even if the patient has normal cardiac biomarker levels and even if the patient does not meet the risk factors for the GRACE score (Rosa A and Jose E, 2013).

Studies on the predictive value of MPV or IPF to determine the prognosis of ACS patients are still contradictory. Several studies observed an increase in mortality in ACS patients with high IPF (11.1\%), compared with patients with high MPV $(5.4 \%)$ or patients with increased TnI at baseline (8.1\%). Another study also observed a trend of high mortality rates in all patients with increased IPF. The increase in IPF is caused by an increased platelet turnover rate which can lead to poor treatment response and disease prognosis.

Positive correlations between mortality and IPF were also observed in non-cardiovascular diseases such as sepsis and disseminated intravascular coagulation (Grove EL et al, 2011).

Immature platelet fraction or immature platelet fragments has long been associated with coronary heart disease. Immature platelets have a larger volume and are more active than more mature platelets. These platelets have greater prothrombotic potential, aggregate more quickly with collagen and have higher intracellular levels of thromboxan A2. These factors will affect the potential for platelet hemostasis which can contribute to thrombus formation (Paramita et al., 2019).

Total occlusion in STEMI reflects a larger thrombus due to platelet activation and turnover. Most cases of acute coronary syndrome occur due to atherosclerotic plaque rupture. This ruptured plaque mostly clogs less than $50 \%$ of the lumen diameter. After endothelial rupture and erosion, the subendothelial matrix is exposed to circulation. This causes platelet adhesion followed by platelet activation and aggregation, which results in thrombus formation. Increased consumption of platelets at atherosclerotic plaque sites results in the release of larger immature platelets into the circulation (Paramita et al., 2019).

Elevated immature platelets are associated with arterial thrombosis, and recent studies have shown that immature platelets are an independent predictor of acute coronary syndrome patients receiving antiplatelet therapy and can be used to stratify risk in acute coronary syndromes. A study conducted by Jiminez et al. demonstrated that increased IPF within the first 24 hours was associated with a poor prognosis even in patients whose GRACE scores were not considered high risk (Paramita et al, 2019).

Platelets play an important role in atherosclerotic plaque formation and thrombus. Platelet activation results in the secretion of molecules such as thromboxane A2 and 5-hydroxytrypta-mine (5HT) which cause vasoconstriction; platelet derived growth factor (PDGF), b-thromboglobulin (b-TG) and platelet factor 4 (PF4) which stimulate arteriosclerosis; leukotrienes that amplify the inflammatory response; and glycoprotein IIb / IIIa receptors which activate the hemotatic system for platelet aggregation and thrombus formation (Huang HL et al., 2019).

Immature Platelet Fraction is also increased in ACS patients. When the patient has increased aggregation and thrombus 
formation, it means that the rate of consumption of mature platelets is very high. To compensate for the loss of platelets, the body operates a positive feedback mechanism to produce more immature platelets with an increased volume. Thus, the platelet count will remain the same in ACS and non-ACS patients, but IPF levels will increase only in ACS patients (Huang HL et al., 2019).

However, several studies have shown that IPF cannot be an independent predictor of the diagnosis of ACS. Platelet activity is known to be related to platelet volume and the presence of immature platelets. It is important to note that not all platelets with increased volume are immature platelets. High immature platelet fraction is generally caused by the rapid response of megakaryocytes, which produce immature platelets as compensation to compensate for rapid platelet loss. In acute coronary syndrome patients, there is a stable consumption of platelets caused by endothelial cell injury and atherosclerotic plaque formation during disease progression. To compensate for the longterm loss of platelets, megakaryocytes are stimulated by thrombopoietin and converted to polyploidy megapytocytes which produce mature platelets with increased volume with a slow response. Thus, IPF alone may not be a good biomarker for identifying acute coronary syndrome patients if it is not combined with other biomarkers (Huang HL et al., 2019).

\section{CONCLUSION}

Based on the research results that have been stated previously, the conclusions that can be drawn in this study are:

1. There is a significant difference in IPF values between patients with acute coronary syndrome who are STEMI and NSTEMI/UAP.

2. The IPF value in STEMI patients was much higher than NSTEMI/UAP patients, namely $6.2 \%(3.5 \%-16.8 \%)$ vs $2.9 \%(0.7 \%-12.0 \%)$.

\section{Acknowledgement: None}

Conflict of Interest: None

\section{Source of Funding: None}

\section{Ethical Approval: Approved}

\section{REFERENCES}

1. Costa SC., Matos Vinagre CGE., Marte Chacra AP., Andrade de Azevedo MR. 2015. Platelet Indices in Patients with Acute Coronary Syndrome. Journal of Biosciences and Medicines ; 3:71-76.

2. DEPKES. 2014. Pusat Data dan Informasi Kementerian Kesehatan RI Situasi Kesehatan Jantung . Jakarta : Kementerian Kesehatan RI.

3. Groove E.L., Mette Hvas A, Kristensen S.D. 2009. Immature Platelet in patients with acute coronary syndrome. Article in Thrombosis and Haemostasis ; 101 : 151156

4. Grove E.L.,HVas A.M, Mortensen S.B.,Larsen S.B.,Kristensen S.D. 2011. Effect of platelet turnover on whole blood platelet aggregation in patients with coronary artery disease. J.Thromb Haemost JTH;9:185e91.

5. Huang HL, Chen CH, Kung CT, et al. 2019. Clinical Utility of Mean Platelet Volume and Immature Platelet Fraction in Acute Coronary Syndrome. Biomedical Journal.42.(2019).107-115.

6. Khalifa KA., Helwa MA.,Mohammed AM. 2017. Reticulated platelets in acute coronary syndrome patients. Menoufia Medical Journal. 880-886

7. Obeidi SR., Ahmedm SH., Obeid FA. 2013. Evaluation of Platelet Indices in Patients with Acute Coronary Syndrome. Mustansiriya Medical Journal ; 12 :58 -64.

8. Paramita P., Nurulita A., Ruland DNP. 2019. Indonesian Journal Of Clinical Pathology and Medical Laboratory “ Analysis of Immature Platelet Fraction and Mean Platelet Volume in Acute Coronary Syndrome Patient". 25(3) : 303-306.

9. Perhimpunan Dokter Spesialis Kardiovaskuler Indonesia.(PERKI) . 2018. Pedoman Tata Laksana Sindrom Koroner Akut. Edisi IV. Jakarta. 
10. Riset Kesehatan Dasar. 2013. Jakarta: Badan Penelitian dan Pengembangan Kesehatan Kementrian Kesehatan RI.

11. Rosa A., Jose R GP, Marcedes SB, Francisco MH., Candido ML, Pedro PO. 2013. Immature Platelet Fraction : A New Prognostic Marker in Acute Coronary Syndrome. Scientific letters/Rev Esp. Cardiol. 2013;66(2)145-154

12. Rubini Gimenez M., Reiter M., Twerenbold R., et al. 2014. Sex specific chest pain characteristics in the early diagnosis of acute myocardial infarction. JAMA Intern Med;174:241-9
13. Pasupathy S., Air T., Dreyer RP., Beltrame JF. 2015. Systematic review of patients presenting with suspected myocardial infarction and non obstructive coronary arteries. Circulation; 131 (10) : 861-870.

How to cite this article: Sari EB, Akbar NZ, Hariman H. Immature platelet fraction (IPF) levels in acute coronary syndrome (ACS) patients. International Journal of Research and Review. 2021; 8(8): 682-688. DOI: https://doi. org/10.52403/ijrr.20210890 